\title{
Method for Estimating of Construction Cost of a Building Based on Previous Experiences
}

\section{Cláudio Ricardo Bettini, Orlando Celso Longo, Luciane Ferreira Alcoforado, Alana Caroline Gamba Maia}

Escola de Engenharia, Universidade Federal Fluminense, Niterói, Brazil

Email: claudio.bettini@gmail.com

How to cite this paper: Bettini, C.R., Longo, O.C., Alcoforado, L.F. and Maia, A.C.G. (2016) Method for Estimating of Construction Cost of a Building Based on Previous Experiences. Open Journal of Civil Engineering, 6, 749-763.

http://dx.doi.org/10.4236/ojce.2016.65060

Received: September 7, 2016

Accepted: December 2, 2016

Published: December 5, 2016

Copyright $\odot 2016$ by authors and Scientific Research Publishing Inc.

This work is licensed under the Creative Commons Attribution International

License (CC BY 4.0).

http://creativecommons.org/licenses/by/4.0/

\begin{abstract}
In a real estate project, the estimated cost of construction and the revenues generally represent together the most important values of its feasibility study. When a decision of undertaking a project is made, often there are few definitions of what is about to be constructed, and frequently not enough to ensure the accuracy of the estimated costs. Considering a global tendency on reducing margins of return over the real estate markets, slight variations of the construction cost can jeopardize the success of the whole real estate enterprise and even the financial stability of the builder or of the developer. This article aims at presenting a method of estimating the building construction costs applicable at the stage of feasibility studies, being able to provide acceptable errors.
\end{abstract}

\section{Keywords}

Real Estate Development, Construction Cost Estimation, Cost Forecast, Cost Estimation Error

\section{Introduction}

The decisions of buying land and carrying out enterprises are crucial for the real estate companies. These decisions must be grounded by dynamic feasibility studies. Small variations in the cost of a construction may endanger the profitability of enterprises. The financial result of a single enterprise can determine the continuity, the growth or even the bankruptcy of a developer or a construction company.

According to Gonçalves (2014) [1], in Brazil, the net margin of real estate enterprises varies between $9 \%$ and $13 \%$ of its revenue (General Sales Value-GSV) while the cost of its construction ranges from $40 \%$ to $50 \%$ of GSV. In a hypothetical situation, $5 \%$ of error on a construction cost estimation, if associated with additional overcosts of other 
$5 \%$ because of inefficiency in the construction process, can take the total variations in the cost of the construction to around $10 \%$, accounting for about $5 \%$ of the revenue (GSV) and eventually involving more than $50 \%$ of the net margin: a very undesirable but yet possible and even common situation.

Considering the urge with which most of real estate investment decisions need to be taken, it is expected that the construction costs estimation can be quickly provided only on the basis of either architectural study or from legal projects even before construction has been fully studied, designed, detailed and specified. Smith (2007) [2] states that:

The primary function of approximate estimating is to produce a forecast of the probable cost of a future Project, before the Building has been designed in detail and contract particulars prepared. In this way the building client is made aware of his likely financial commitments before extensive design work is undertaken.

Estimators have the responsibility and the challenging role of basing the most important business decisions when there is still very little information available.

The present method was based on 30 residential commercial real estate enterprises, constructed between the years of 2004 and 2015 by the same construction and development company in the metropolitan area of Rio de Janeiro. The method combines elements of parametric and analogical estimation techniques, always providing more accurate estimates than purely the parametric or analogical methods. This method aims at fulfilling the need of accuracy and swiftness in the construction cost estimation when very little information is available. The method requires some previous construction experience to start the databank of organized information and evolves with the increase of experience.

\section{Methodology}

The first step is the definition of a general Work Breakdown Structure (WBS) able to properly account for and compare the construction costs of all the company's projects. Here, twenty five out of the thirty constructions were randomly selected to test the method and the remaining was set aside to finally validate its accuracy. The first twenty five were divided into five groups ( $A, B, C, D$ and $E$ ) considering their similarities under following criteria:

a) Usage: Residentialor Commercial;

b) Size (Gross Building Area): under 10,000 $\mathrm{m}^{2}$, around 15,000 $\mathrm{m}^{2}$, around 20,000 $\mathrm{m}^{2}$ and over;

c) Class of Specification: A (superior), B (medium), C (basic). In Brazil, real estate market buildings are usually very similar in respect to its adopted construction technology and process adopted. Besides the building design, what differentiates one from another are usually just some finishing materials like frames (basically can be of wood or aluminium), pavement (basically can be of porcelain or ceramic) and some appliances like toilets, washbasin and water taps;

d) Form: Small Towers (at least 10 storeys), Medium Towers (at least 16 storeys) and High Towers (more than 16 storeys). 
Group A got 6 residential constructions with areas around 20,000 $\mathrm{m}^{2}$, Specification Class "C" and Small Towers; Group B got 3 residential constructions with areas over 20,000 $\mathrm{m}^{2}$, Specification Class "A" and Small Towers; Group C got 4 residential constructions with areas around 15,000 $\mathrm{m}^{2}$, Specification Class " $\mathrm{B}$ " and "C" and Medium Towers; Group D got 6 residential constructions with areas around $15,000 \mathrm{~m}^{2}$, Specification Class "A" and "B" and High Towers; Group E got 6 commercial constructions with real areas up to $10,000 \mathrm{~m}^{2}$, Specification Class " $\mathrm{A}$ " and "B" and Small Towers.

Whatever the grouping criterion is, it must point to the homogeneity of the average cost per square meter. The more projects there are available, the more groups can be created, thus enhancing precision.

\subsection{Definition of the Acceptable Margin of Error}

Although estimations always carry associated margins of error, it is still a common practice among the Brazilian real estate companies not to consider any error at all. In the academy, acceptable margins range from $\pm 10 \%$ to $\pm 5 \%$, regardless of the level of detail of the projects and despite the information that can be available. According to Halpin (2004) [3], still in the preliminary phases of design, an error up to $10 \%$ would be totally acceptable. Limmer (1997) [4], on the other hand, says that the percentage of error of any estimation will never be lower than $5 \%$, even if it is fully detailed as specified.

Despite those academic understandings, we all know that cost estimations with margins of error beyond 5\% are no longer tolerable by the real estate market, due to its reduced gross margins, sometimes very close to $5 \%$ of the GSV. It is also a common sense among the market, by experience, that projects with a lot of design repetition or highly standardized construction processes can enable errors lower than $5 \%$ even in preliminary study stages. Morton (1995) [5] corroborates this idea when he says: “...a really accurate estimate of a building's cost cannot be made until the details of the design are known-except in the case of standard repeated building types". For instance, in this article the error obtained while comparing the actual cost (indexed) performed on the real constructions and a current estimation with the present method based only on the legal project remained always between $\pm 2 \%$, as we will demonstrate further. Gonçalves (2014) [1] states that by the year 2004 the errors of the cost estimations at Inpar, a wellknown Brazilian real estate company, did not exceed $2 \%$ when compared with the final budgets.

The error of a given estimation can be found in two ways: 1) by comparing the estimation with the final budget (based on the executive design) or 2) by comparing the estimation with the actual costs incurred by the end of the construction. Although the first criterion is a usual procedure, once it protects the estimator, exempting him from the eventual errors of the construction process, for the entrepreneur only the second criterion matters. The project only makes sense if it is advantageous altogether. Design teams, managers, estimators, quantity surveyors, planners and construction team, everyone, must work aligned and subordinated to the same vision and command as an in- 
tegrated team, committed to the same goals, pursuing the fulfillment of the project requirements, respecting the guidelines and assumptions set out at the feasibility studies. Adopting a composite margin of $2 \%$ for the first criterion, and another $2 \%$ for the eventualities of the construction process, we come out with $4 \%$ of total acceptable error, satisfying the second criterion, and margins seem to be at the same time achievable, fair and enough to satisfy the feasibility restrictions.

\subsection{The Need for Indexing the Values of the Study}

The life cycle of a real estate development project, from the land acquisition to the delivery of the built units, usually takes a great time span and can last three, four or even more years. This fact demands that the construction cost be indexed from the estimate through the construction. Values can be transformed into either an index or a hard currency, in order to attenuate the effects of inflation. Owing to the considerable time span, this transformation must be done even in countries where there is low inflation.

In the present study, all values have been indexed by the National Civil Construction Index (INCC), a Brazilian official index, published monthly by Fundação Getúlio Vargas (FGV). The INCC index was chosen due to two reasons: 1) in the Brazilian real estate market it is common to update the revenues with the INCC during the construction period and it seems to be very convenient to keep cash flow inputs and outputs on the same basis; and 2) in this particular situation, considering the studied projects, INCC has been the official index that showed the cost variations most similar to those effectively observed by the company during the same period.

Nevertheless, over the years, the indexing artifice may not be always fully capable to express the real changes in the actual construction costs. Actually, no index is able to perfectly capture and represent the cost variations of a singular and specific company. Therefore, it can be necessary to apply a Monetary Correction Factor (MCF) to correct the differences. Indeed, the detachment between the variations of the actual costs observed in the studied company and the variations on the INCC-FGV during the same period was about $1.00 \%$ y.a.

Professor João da Rocha Lima Jr. [6], from the Real Estate Center of the Polytechnic School of the University of São Paulo (NRE-Poli) writing on the Carta NRE-Poli of April to June 2014, corroborates with this idea when he declares that through the years of 2005 to 2013, distortions on the cost of the supplies, specially the cost of subcontracted workforce and costs related to loss of productivity and urgencies during this period were not captured by the INCC index. And he suggests that, starting on the year of 2005 , an additional variation of $3.50 \%$ y.a should be added to the INCC variation in order to compensate the real inflation suffered by the construction sector during this period. Although the professor observed a significantly higher detachment, it comes out that the detachment really occurs, and sometimes must be mitigated.

\subsection{The Equivalent Area and Equivalent Cost Concepts}

Every square meter of a building can be associated with a virtual area: its equivalent 
area, which expresses its cost of construction in relation to the cost of a reference area, a specific area taken as a universal comparison pattern.

As an illustration, let us take two areas of a residential building: a living room with 20 $\mathrm{m}^{2}$ and a terrace (uncovered floor) with $20 \mathrm{~m}^{2}$. By hypothesis, after checking all the costs needed for constructing the living room, we come up to $\mathrm{R} \$ 22,000.00$, or $\mathrm{R} \$ 1100.00 / \mathrm{m}^{2}$. In this situation, $\mathrm{R} \$ 1100.00 / \mathrm{m}^{2}$ represents the equivalent cost of the area. Similarly, we find the cost to build the terrace at $\mathrm{R} \$ 15,000.00$, and $\mathrm{R} \$ 750.00 / \mathrm{m}^{2}$. Considering the cost of a standard reference area as $\mathrm{R} \$ 1000.00 / \mathrm{m}^{2}$, we can assign to the living room an equivalence ratio of $110 \%(\mathrm{R} \$ 1100.00 / \mathrm{R} \$ 1000.00)$ and to terrace $75 \%(\mathrm{R} \$ 750.00 / \mathrm{R} \$ 1000.00)$. The equivalent area of the living room will be equal to $22 \mathrm{~m}^{2}(20 \mathrm{~m} \times 110 \%)$, while the terrace's will be equivalent to $15 \mathrm{~m}^{2}(20 \mathrm{~m} \times 75 \%)$. Although the two areas have the same real area $\left(20 \mathrm{~m}^{2}\right)$, they both have different costs and hence different equivalent areas.

If every typical area is well defined in terms of its cost, we can build a generic table (like Table 1) where each typical area is associated to an equivalent area and consequently to an equivalent cost coefficient. The level of detailing of the classification will be different for each company, due to its experience, knowledge and due to the best balance between the maximum desired levels of complexity and the need of accuracy of the estimation. The Total Equivalent Area (TEA) of a given project will be obtained by the sum of all different areas of the construction multiplied by their respective equivalence coefficient.

In Brazil, the real estate market is mainly represented by small-sized or mediumsized companies that operate in specific market niches generally developing very similar projects in terms of design and in terms of the adopted construction processes. The most similar the constructions are, the most significant and representative the concept of equivalent area will be.

\subsection{Parts of the Construction Cost}

The equivalent area and equivalent cost concepts are highly effective analogy methods to estimate the parts of the costs of a construction that can be more easily associated to the "physical" areas of the construction. The remaining parts of the cost are more effectively estimated parametrically. Estimating the Total Construction Cost (TCC) should be done in parts, as shown in Equation (1):

$$
\mathrm{TCC}=\mathrm{EAC}+\mathrm{ADMC}+\mathrm{EXC}
$$

The Equivalent Area Cost (EAC) is the portion that can aggregate all the costs of the construction that can be directly associated to real areas of construction. The costs included in the EAC portion must be always common among the other projects of the same group of similarity;

The Administration Cost (ADMC) is the portion that can be directly associated to the administrative staff. This portion varies mostly with the time span of the construction, and not with its total equivalent area.

The Extra Costs (EXC) is the portion of the cost that cannot be easily associated to 
Table 1. Cost equivalence.

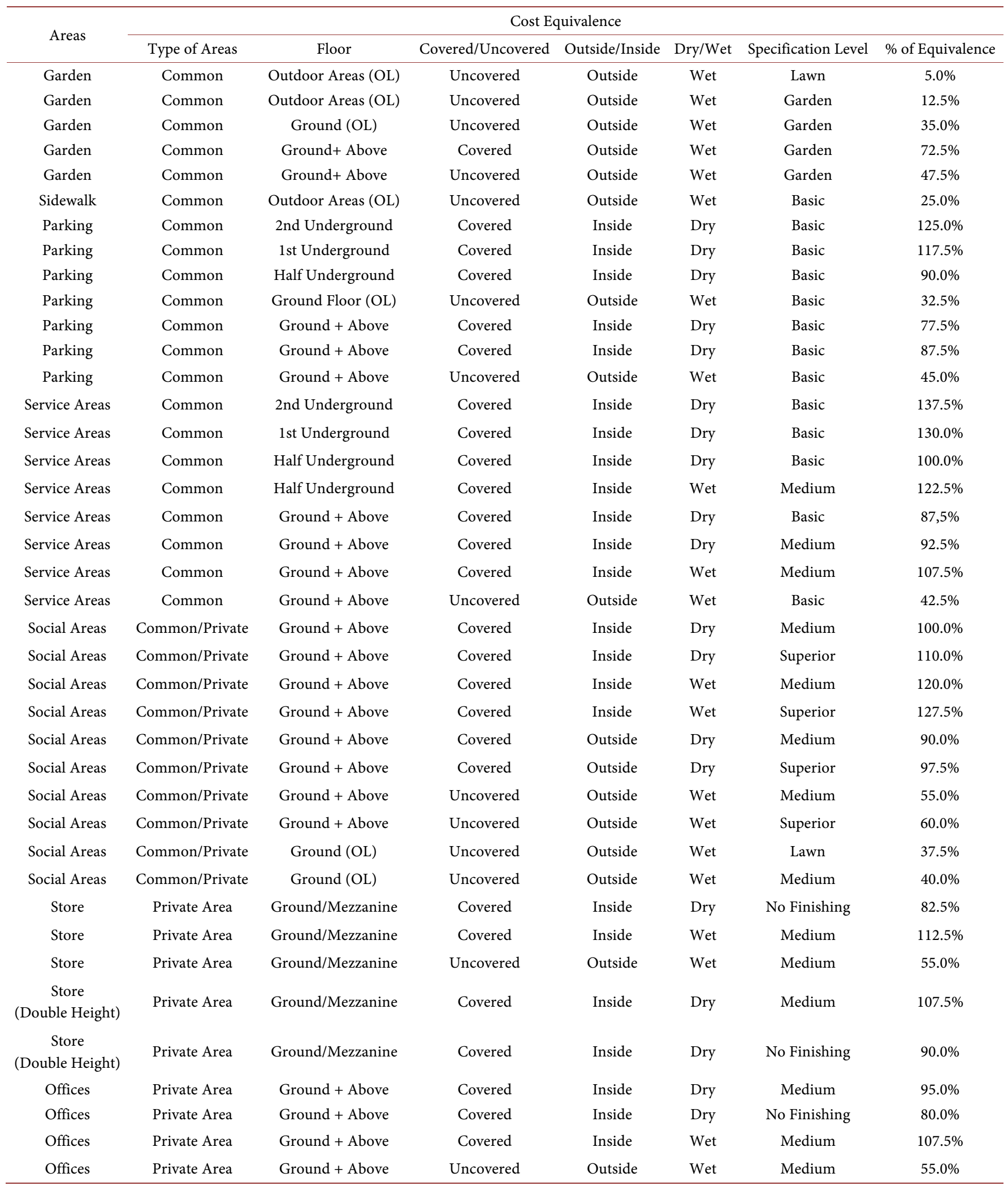

a. Source: Author. 
EAC or ADMC. These are the costs that represent very specific characteristics and particularities of the project under study.

\subsubsection{Average Equivalent Area Cost}

Once we have determined the EAC and the TEA of a construction, we can divide one by the other (EAC/TEA) to find the Average Equivalent Area Cost (AEAC). Taking the sum of the EAC of all finished constructions of a same group and dividing it by the sum of the TEA of the respective constructions we get the Average Equivalent Area Cost (AEAC) of the group. The AEAC is the heart of the method, responsible for about $87 \%$ of the estimation. The remaining $13 \%$ of the total cost of the construction will be easily estimated through the ADMC and EXC portions, using very simple parametric methods.

\subsubsection{The Equivalent Area Cost Calculation}

The EAC is given by the product of the total equivalent area of a construction (TEA) and the cost of the average equivalent area cost (AEAC), as presented in Equation (2), as follows:

$$
\mathrm{EAC}=\mathrm{TEA} \times \mathrm{AEAC}
$$

AEAC deals with similarity. Whenever the project presents particularities that distinguish it from the others of a same similarity group, a Cost Equalization Factor (CEF), must be applied to the AEAC in order to correct it, as given by Equation (3), as follows:

$$
\mathrm{CEF}=(1+\mathrm{Fe}+\mathrm{Fr}+\mathrm{Fh}+\mathrm{Fb}+\mathrm{Ff}+\mathrm{Fc})
$$

On Equation (3):

a) Fe is a scale factor. The bigger the construction is, the bigger the bargaining power of the constructor towards the suppliers. Fe is measured by the percentage difference between TEA of the construction under study and the average TEA of the reference constructions of the same similarity group.

b) Fr is a floor repetition factor. The more repeated floors there are, the lower the construction costs per area will be. Fr is measured by the percentage difference between the rate of repetition of the study and the average repetition rate of the reference constructions of the same similarity group.

c) Fh is a factor related to the height of the construction. The higher (or deeper) the construction is, more vertical displacement or tasks sequencing the construction will need, and consequently the higher will be the construction costs per area.

d) $\mathrm{Fb}$ is a factor related to the number of blocks. The more segmented into blocks the construction is, the higher will be the construction costs per area.

e) Ff is a factor associated with both the perimeter of the building and the shape of its facades. According to Morton (1995) [5]: "It is obvious that the perimeter of a building of a given area will be a different length depending on the plan shape; and consequently the cost of those elements which are directly related to perimeter length such as walls will also vary with plan shape." The Ff establishes a relationship between the construc- 
tion area and the area of its facades. The higher this index gets, the more expensive the construction will be. Ff is given by the ratio between the perimeter of a given pavement and the perimeter of a circle of the same area of this same pavement. The calculation of Ff can consider all the building floors, weighted by their respective areas, and more simply, it could just consider the typical storey, which is repeated more often.

f) $\mathrm{Fc}$ is a factor associated with the class of the building with respect to its general finishing. The more expensive the finishing is, the higher the construction costs per area will be. Fc considers the internal and external cladding, window frames and other finishing items, that cannot be captured by the other factors. $\mathrm{Fc}$ is more subjective than the other factors, especially considering the degree of uncertainties associated to the finishings of the constructions under study.

The variations and limits of the components of CEF depend on each company's reality, including construction processes, adopted technologies and general specifications of its constructions. In this work they were empirically defined based on the experience over the 30 chosen projects, as follows:

a) Fe: For every $\pm 10 \%$ from the average, sum up or subtract, respectively $0.50 \%$ of the factor, limited to $2.50 \%$.

b) Fr: For every $\pm 10 \%$ from the average, sum up or subtract, respectively, $0.35 \%$ of the factor, limited to $3.50 \%$. Above $100 \%$ of repetition the potential for cost reduction decreases to a point where it can be ignored.

c) Fh: Each additional or reductional pavement of the construction, when compared to the average, must be respectively, added or subtracted, of $0.15 \%$, limited to $1.00 \%$.

d) $\mathrm{Fb}$ : Each additional or reductional block of the construction, when compared to the average, must be respectively, added or subtracted, of $0.15 \%$, limited to $1.00 \%$.

e) Ff: For every $\pm 10 \%$ from the average, sum up or subtract, respectively, $0.35 \%$ of the factor, limited to $3.00 \%$. The perimeter of the floor was considered by its external walls, excluding the balconies.

f) Fa was limited to $4.00 \%$.

Beyond these limits the construction cannot be considered so similar to a reference group, and the estimation will probably deliver an error larger than acceptable.

It is evident that the CEF cannot enclose all the possibilities that modify the cost of a building, but it surely encloses the ones that represent the most significant differences between different projects, and it is enough to ensure the expected level of accuracy. Many other factors could have been defined. It is important, however, not to lose sight of the simple and expeditious character that estimation must remain.

Table 2 presents a resume of how the CEF components affect each cost item of the constructions of a similarity group. Table 2 was based on a Work Breakdown Structure (WBS), common to all the projects. The groups of the WBS are the same used for the budgeting process, and the maximum, the minimum and the mean percentage contributions of each cost item was identified within each similarity group. The factors that form CEF have their limits defined by the total influence they can have over the entire cost, considering their influence over each cost group of the WBS. The limit boundaries 
Table 2. Factors that correct the cost.

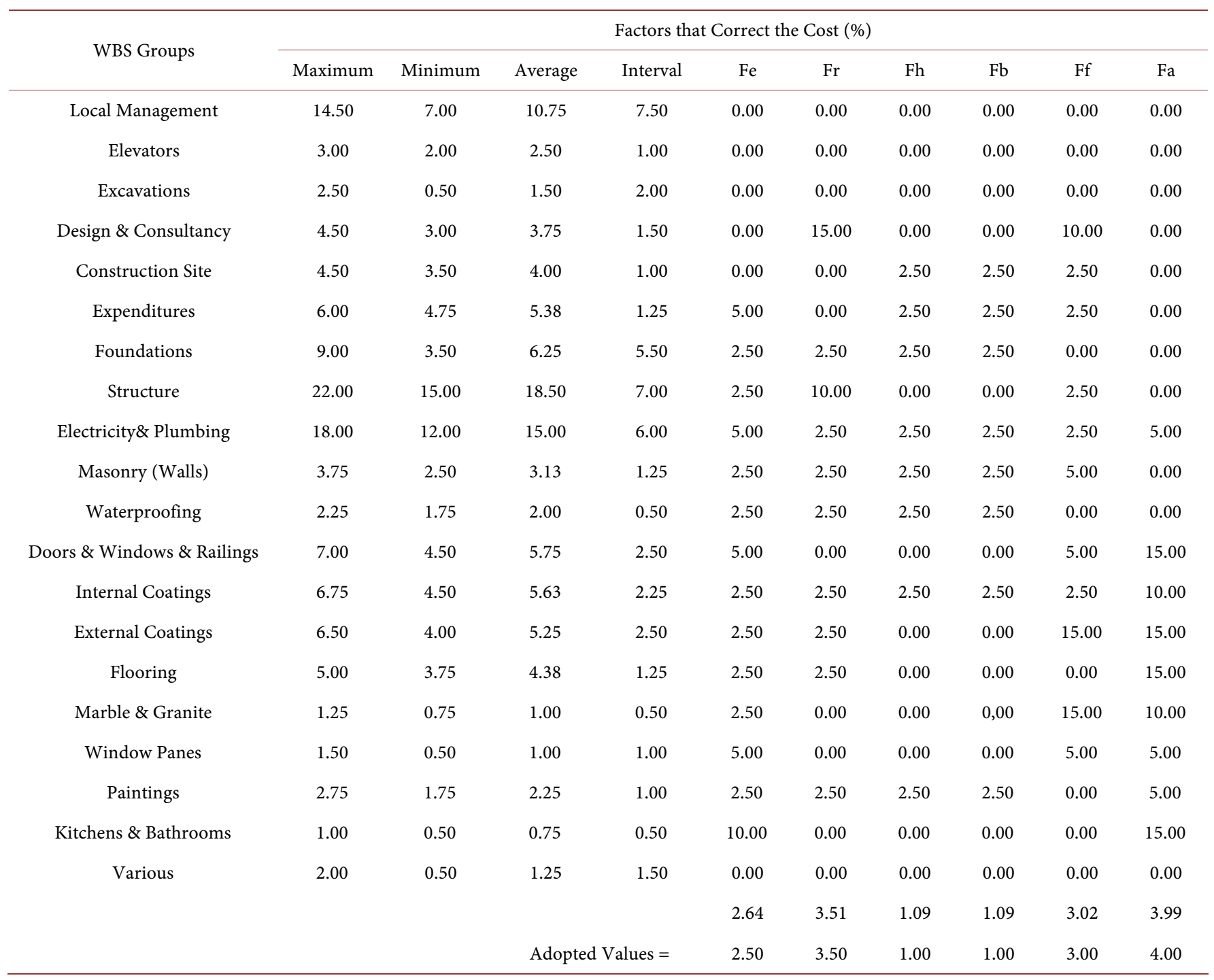

a. Source: Author.

of each component of the CEF factor were verified by adding the product that each factor can have over the average contribution of each group. The variations and limits for the components of CEF were defined empirically in this work. Using linear regression, however, it may be possible to find more precise results.

The first three groups of the WBS (Local Management, Elevators and Excavations) were not considered, once they are fully calculated through the ADMC and EXC portions.

When creating the groups, data base CEF is applied to each construction in order to bring its cost closer to the average, counteracting its particularities. Otherwise, when estimating the cost of a new construction CEF is applied to point out its particularities exposing its differences in order to adjust the correct cost.

Applying CEF and MCF factors, the equation of the EAC portion becomes: 


$$
\mathrm{EAC}=\mathrm{TEA} \times \mathrm{AEAC} \times(1+\mathrm{MCF}) \times \mathrm{CEF}
$$

or:

$$
\mathrm{EAC}=\mathrm{TEA} \times \mathrm{AEAC} \times(1+\mathrm{MCF}) \times(1+\mathrm{Fe}+\mathrm{Fr}+\mathrm{Fh}+\mathrm{Fb}+\mathrm{F}+\mathrm{Fc})
$$

The ADMC and the EXC portions do not need to be corrected by the MCF because its estimation is done at current values.

\subsubsection{The Administration Cost Calculation}

The ADMC cost is a function of the Time Span (TS) of the construction and the average monthly cost of the Management Team (MT), according to Equation (6), as follows:

$$
\mathrm{ADMC}=\mathrm{TS} \times \mathrm{MT}
$$

The way of estimating TS and MT parameters depends on the complexity of the construction. The simplest way to do this it to take the average values of the reference group. But it is also fast, easy, and more precise to estimate it using the following tools.

With a PERT chart consisting only of the most critical working groups of a typical construction of the group, as illustrated in Figure 1, it is possible to calibrate the effect of great magnitude events that can reduce or amplify the total time span of the project under study. With a spreadsheet, as illustrated in Table 3, the management team can be designed for the period of the construction. Summing up the wages and costs associated to each professional (social charges, benefits, food, transportation, uniforms, personal protective equipment and tests) we get the total cost of the management team and in

\begin{tabular}{|c|c|c|c|c|c|c|c|c|c|c|c|c|c|}
\hline Management Staff & \multicolumn{13}{|c|}{ Management Team Distribution (months) } \\
\hline Engineer/General Manager & 12 & 1 & 1 & 1 & 1 & 1 & 1 & 1 & 1 & 1 & 1 & 1 & 1 \\
\hline Intern & 32 & 2 & 2 & 2 & 2 & 3 & 3 & 3 & 3 & 3 & 3 & 3 & 3 \\
\hline Clerk Of Works & 12 & 1 & 1 & 1 & 1 & 1 & 1 & 1 & 1 & 1 & 1 & 1 & 1 \\
\hline Site Chief & 12 & 1 & 1 & 1 & 1 & 1 & 1 & 1 & 1 & 1 & 1 & 1 & 1 \\
\hline Warehouse Keeper & 12 & 1 & 1 & 1 & 1 & 1 & 1 & 1 & 1 & 1 & 1 & 1 & 1 \\
\hline Office Boy & 11 & & 1 & 1 & 1 & 1 & 1 & 1 & 1 & 1 & 1 & 1 & 1 \\
\hline Gatekeeper & 24 & 2 & 2 & 2 & 2 & 2 & 2 & 2 & 2 & 2 & 2 & 2 & 2 \\
\hline Night Gatekeeper & 12 & 1 & 1 & 1 & 1 & 1 & 1 & 1 & 1 & 1 & 1 & 1 & 1 \\
\hline
\end{tabular}
sequence the average monthly cost of the management team (MT).

Table 3. Management team distribution.

a. Source: Author. 


\begin{tabular}{|c|c|c|c|c|c|c|c|c|c|c|c|c|c|c|c|c|c|c|c|c|c|c|c|c|c|}
\hline & Nome da tarefa & 11 & Duration $\mathrm{r}$ & Predecessors ${ }^{\top}$ & M-1 & M1 & M2 & M3 & M4 & M5 & M6 & M7 & M8 & M9 & M10 & M11 M12 & M13 & M14 M15 & M16 I & M17 M18 & M19 & M20 & M21 M22 & M23 & M24 \\
\hline \multicolumn{26}{|l|}{1} \\
\hline 2 & $\triangle$ BUILDING & & 520 days & & & & & & & & & & & & & & & & & & & & & & \\
\hline 3 & FOUNDATIONS & & 7 months & & & & & & & & & 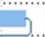 & & & & & & & & & & & & & \\
\hline 4 & STRUCTURE & & 7 months & 3FS-1month & & & & & & & + & & & & & $\ldots$ & & & & & & & & & \\
\hline 5 & MANSORY & & 5 months & 4SS-3months & & & & & & & c & & & $\rightarrow$ & & & & & & & & & & & \\
\hline 6 & FACADE & & 6 months & 5 & & & & & & & & & & & & & & & & & $\exists$. & & & & \\
\hline 7 & COMMON FLOORS & & 4 months & 6FS-1month & & & & & & & & & & & & & & & & 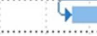 & & & & & \\
\hline 8 & TERMINATION & & 3 months & 7 & & & & & & & & & & & & & & & & & & & & & \\
\hline
\end{tabular}

Figure 1. The simplified schedule. Source: Author.

The design of the management team depends on several factors of each construction and should be done case to case. However, average values could also be used. Here, the considered management team includes professionals that work on the construction site and that perform management positions or are associated to indirect costs of the construction, as illustrated in Table 3.

The ADMC portion typically ranges around $10 \%$ of the TCC. The bigger the construction is, the smaller the ADMC portion percentually tends to be, once the cost of administration tends to be more diluted or apportioned within the total cost. For example, one same engineer can be alone responsible for the construction of $10,000 \mathrm{~m}^{2}$ or $20,000 \mathrm{~m}^{2}$. Over the second case the weight of his cost will be half as over the first.

\subsubsection{The Extra Costs Calculation}

The EXC portion covers all costs that could not be considered in the previous portions. The EXC should not weigh more than 3\% over the TCC. Each component of the EXC portion can be estimated parametrically based on previous construction experiences. When no previous work is available to support a parameterization, it will be necessary to budget it. Some examples of extra costs are: special costs of foundations; groundwater drawdown systems; pools and leisure equipment; mechanic garage ramps; power generators, power and network street extensions; central heating; treatment and reuse of water; solar energy systems; automation systems; elevators; furniture and appliances; and other particular costs. Elevators, for example, were present in all the 30 projects that based this article. Nevertheless, as they cannot be directly associated to a real area, they must be considered in the EXC portion.

\section{Validation of the Method}

The five projects separated to validate the method were estimated, retrospectively, using the method. At this point, these projects were fully constructed having their costs completely incurred. Thus the estimated error could be precisely evaluated.

In the five validation estimates the TS parameter was based on the typical PERT diagram of the respective reference groups, while the MT parameters were obtained using management team distribution spreadsheets as shown in Table 3. The factors that comprised each CEF were calculated considering the particularities of each of the five projects. For the estimation number 1, AEAC was taken as the average AEAC value of the group of reference and the MCF was considered as $1.00 \%$ y.a., according to what was previously observed. On the estimations number 2 to 5 , AEAC was taken, in each 
case, as the AEAC of the most similar project of each group of reference. MCF was also always considered as $1.00 \%$ y.a.. AEAC deals mainly with similarity, so it can be taken as a simple or weighted average of a selected number of previous projects chosen on purpose, or form only one project as long as it is very similar. Table 6, supported by Table 4 and by Table 5, presents a summary of the five estimations with the error obtained between these estimates and the real costs incurred.

\section{Analysis of Results}

Within the five projects used for testing the method the real errors of the estimations were never beyond the interval of $\pm 2 \%$, as Table 6 demonstrates. In fact, 4 out of 5 results were between $+0.59 \%$ and $-0.61 \%$, being even more accurate than expected.

Tables 7-10 show how undesirable results with great variability we would get if we only related areas (real or equivalent) to costs per square meter (real or equivalent) as done traditionally in so many companies.

Table 4. Groups of reference data.

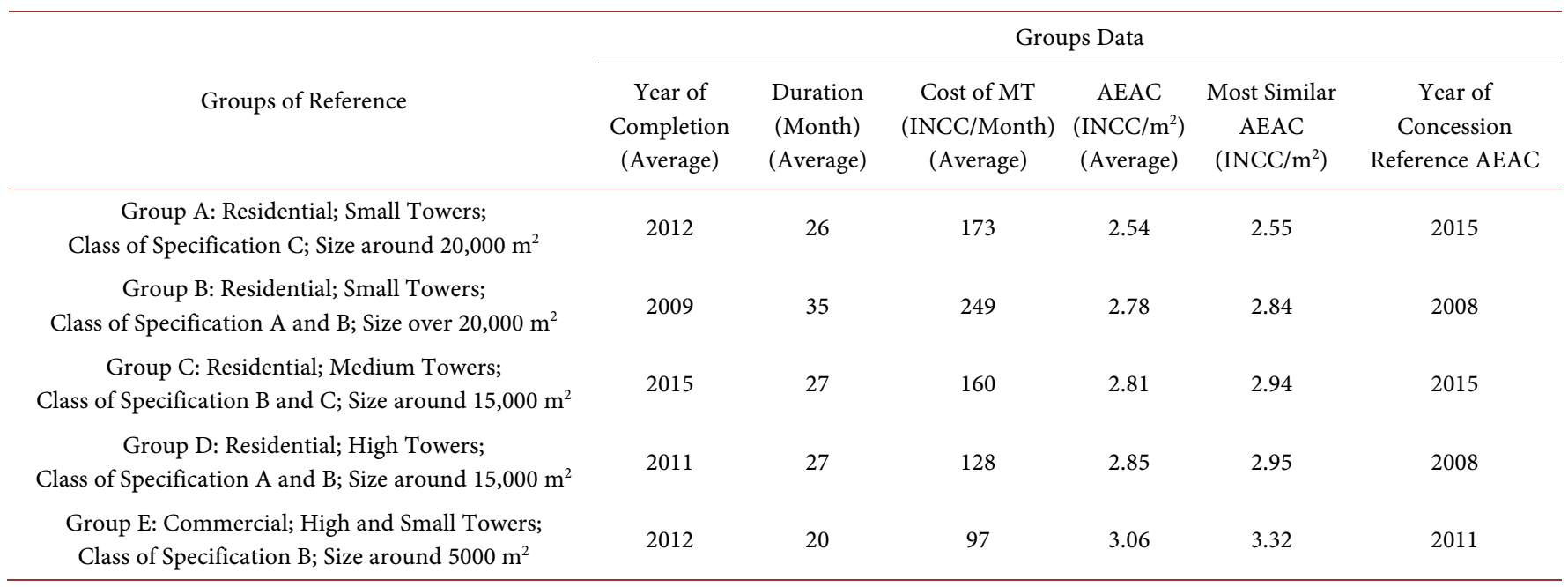

a. Source: Author.

Table 5. Estimated constructions data.

\begin{tabular}{|c|c|c|c|c|c|c|c|}
\hline \multirow[b]{2}{*}{$\begin{array}{c}\text { Estimated } \\
\text { Constructions }\end{array}$} & \multicolumn{6}{|c|}{ Estimated Constructions Data } & \multirow[b]{2}{*}{$\begin{array}{c}\text { Adopted } \\
\text { AEAC } \\
\left(\mathrm{INCC} / \mathrm{m}^{2}\right)\end{array}$} \\
\hline & $\begin{array}{l}\text { TEA } \\
\left(\mathrm{m}^{2}\right)\end{array}$ & $\begin{array}{c}\text { Adopted } \\
\text { TS }\end{array}$ & $\begin{array}{c}\text { Adopted } \\
\text { MT }\end{array}$ & $\begin{array}{l}\text { AEAC of } \\
\text { Reference } \\
\left(\mathrm{INCC} / \mathrm{m}^{2}\right)\end{array}$ & $\mathrm{CEF}$ & $\begin{array}{c}\mathrm{MCF} \\
(\%)\end{array}$ & \\
\hline 1: Residential; Small Towers; Class of Specification C & 13.09801 & 25 & 180 & 2.54 & 0.98 & 3.0 & 2.56 \\
\hline 2: Residential; Small Towers; Class of Specification A & 36.56048 & 36 & 340 & 2.84 & 0.98 & -1.0 & 2.75 \\
\hline 3: Residential; Medium Towers; Class of Specification B & 11.20166 & 24 & 180 & 2.94 & 0.98 & 0.0 & 2.90 \\
\hline 4: Residential; High Towers; Class of Specification B & 17.21079 & 28 & 170 & 2.95 & 1.01 & -3.0 & 2.87 \\
\hline 5: Commercial; High Towers; Class of Specification B & 3.88490 & 21 & 80 & 3.32 & 0.99 & -1.0 & 3.26 \\
\hline
\end{tabular}

a. Source: Author. 
Table 6. Validation of the method.

\begin{tabular}{ccccccc}
\hline \multirow{2}{*}{$\begin{array}{c}\text { Estimated } \\
\text { Constructions }\end{array}$} & \multicolumn{5}{c}{ Estimated Constructions Data } \\
\cline { 2 - 7 } & EAC (INCC) & ADMC (INCC) & EXC (INCC) & Estimated (TCC) & Total Cost Incurred (INCC) & Error of the Estimation (\%) \\
\hline 1 & 33.59206 & 4.50000 & 1.17500 & 39.26706 & 39.88254 & $-1.57 \%$ \\
2 & 100.51983 & 12.24000 & 24.19000 & 136.94983 & 137.78187 & $-0.61 \%$ \\
3 & 32.44961 & 4.32000 & 0.82000 & 37.58961 & 37.77413 & $-0.49 \%$ \\
4 & 49.46099 & 4.76000 & 1.35500 & 55.57599 & 55.24855 & $0.59 \%$ \\
5 & 12.66566 & 1.68000 & 0.73500 & 15.08066 & 15.01165 & $0.46 \%$ \\
\hline
\end{tabular}

a. Source: Author.

Table 7. Estimation based on direct relation to average cost of equivalent area.

\begin{tabular}{cccccc}
\hline \multirow{2}{*}{$\begin{array}{c}\text { Estimated } \\
\text { Constructions }\end{array}$} & TEA $\left(\mathrm{m}^{2}\right)$ & $\begin{array}{c}\text { Coference Group Average Cost } \\
\text { (AEAC) }\left(\mathrm{INCC} / \mathrm{m}^{2}\right)\end{array}$ & $\begin{array}{c}\text { Estimated } \\
\text { TCC (INCC) }\end{array}$ & $\begin{array}{c}\text { Total Cost } \\
\text { Incurred (INCC) }\end{array}$ & $\begin{array}{c}\text { Estimation } \\
\text { Deviation (\%) }\end{array}$ \\
\hline 1 & 13.09801 & 2.78 & 36.39547 & 39.88254 & $-9.6 \%$ \\
2 & 36.56048 & 2.89 & 105.56276 & 137.78187 & $-30.5 \%$ \\
3 & 11.20166 & 3.06 & 34.32533 & 37.77413 & 55.24855 \\
4 & 17.21079 & 3.07 & 52.80905 & 15.01165 & $-10.0 \%$ \\
\hline
\end{tabular}

a. Source: Author.

Table 8. Estimation based on direct relation to average cost of real area.

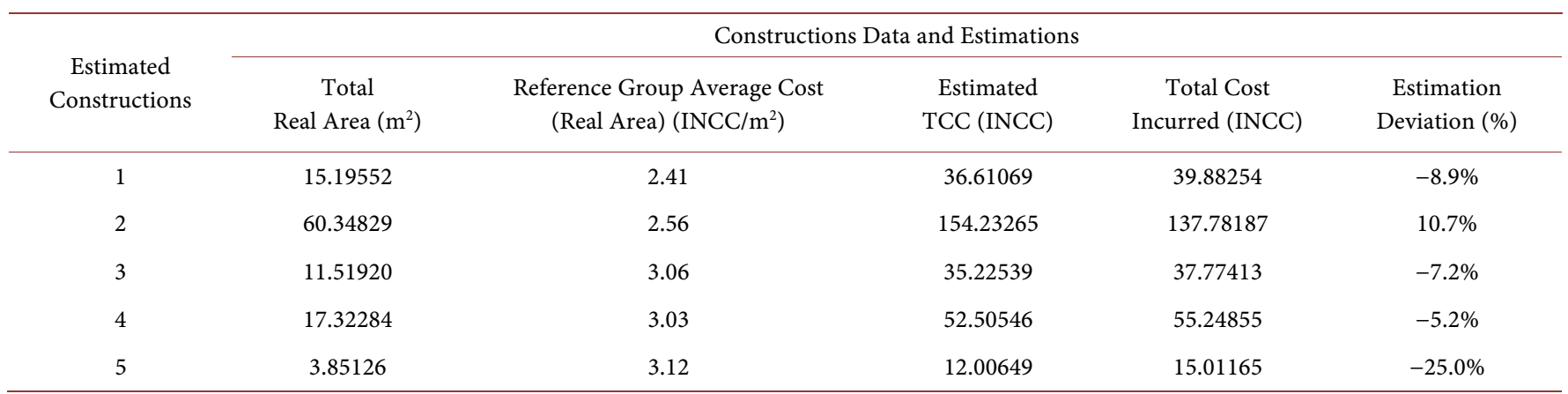

a. Source: Author.

Table 9. Estimation based on direct relation to most similar cost of equivalent area.

\begin{tabular}{|c|c|c|c|c|c|}
\hline $\begin{array}{c}\text { Estimated } \\
\text { Constructions }\end{array}$ & \multicolumn{5}{|c|}{ Constructions Data and Estimations } \\
\hline 2 & 36.56048 & 2.86 & 104.38807 & 137.78187 & $-32.0 \%$ \\
\hline 3 & 11.20166 & 3.23 & 36.21408 & 37.77413 & $-4.3 \%$ \\
\hline 5 & 3.88490 & 3.83 & 14.87726 & 15.01165 & $-0.9 \%$ \\
\hline
\end{tabular}

a. Source: Author. 
Table 10. Estimation based on direct relation to most similar cost of real area.

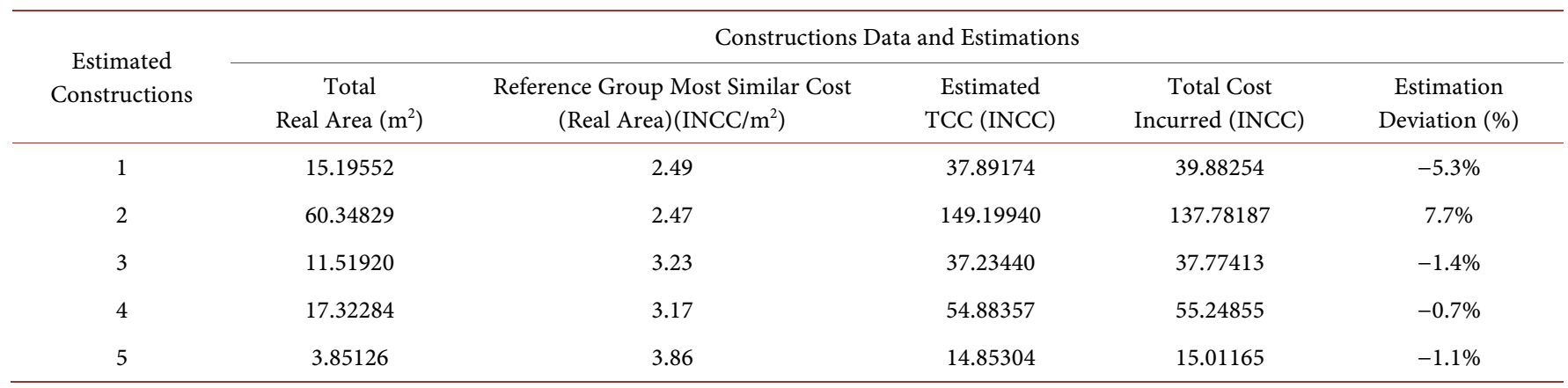

a. Source: Author.

While the error of method in all cases remained limited within the interval of $+0.59 \%$ to $-1.57 \%$, the traditional direct relation estimates showed a great variation ranging from $+10.70 \%$ to $-32.0 \%$, far beyond what would be acceptable by the industry. We can even guess that even where the traditional estimates provide more accurate values they probably do it by chance.

\section{Conclusions}

Using this method, companies with a significant history of completed constructions, with correctly recorded information of construction costs and specifications are fully capable of obtaining very accurate estimates with errors in the limited range of plus and minus $2 \%$.

The method is more consistent and coherent than the traditional direct area comparison between real or equivalent areas, since it considers the particularities of the reference projects as well as the ones of the project under study. Considering the ease of applying this method, and the risks involved, the use of direct area comparison becomes, from now on, almost always unjustifiable.

As a last observation it could be said that neural networks seem to have a great potential to improve this work, turning the estimation process at the same time quicker, more reliable and even more precise.

\section{References}

[1] Gonçalves, C.M. and Ceotto, L.H. (2014) Custo sem susto: Um método para gestão do custo de edificações. 1st Edition, Nome da Rosa, São Paulo.

[2] Smith, J. and Jaggar, D. (2007) Building Cost Planning for the Design Team. 2nd Edition, Elsevier, Queensland.

[3] Halpin, D.W. and Woodhead, R.W. (1998) Construction Management. 2nd Edition, John Wiley \& Sons Ltd., New York.

[4] Limmer, C.V. (1997) Planejamento, Orçamentação e Controle de Projetos e obras. 1st Edition, LTC, Rio de Janeiro.

[5] Morton, R. and Jaggar, D. (1995) Design and the Economics of Building. 1st Edition, Spon Press, London. http://dx.doi.org/10.4324/9780203400364

[6] Lima, J.R.J. (2014) Lições Sobre Bolhas. Carta NRE-POLI abril-junho no. 36-14, São Paulo. 


\section{Abbreviations}

ADMC-Administration Cost

AEAC-Average Equivalent Area Cost

$\mathrm{CEF}$-Cost Equalization Factor

EAC_Equivalent Area Cost

EXC-Extra Costs

FGV_Fundação Getúlio Vargas

GSV-General Sales Value

INCC-Índice Nacional da Construção Civil: National Civil Construction Index

MCF-Monetary Correction Factor

MT-Management Team

PERT_Program Evaluation Review Technique

TCC-Total Construction Cost

TEA-Total Equivalent Area

TS-Time Span

WBS-Work Breakdown Structure

Submit or recommend next manuscript to SCIRP and we will provide best service for you:

Accepting pre-submission inquiries through Email, Facebook, LinkedIn, Twitter, etc. A wide selection of journals (inclusive of 9 subjects, more than 200 journals)

Providing 24-hour high-quality service

User-friendly online submission system

Fair and swift peer-review system

Efficient typesetting and proofreading procedure

Display of the result of downloads and visits, as well as the number of cited articles

Maximum dissemination of your research work

Submit your manuscript at: http://papersubmission.scirp.org/

Or contact ojce@scirp.org 